\title{
Baseline new bone formation does not predict bone loss in ankylosing spondylitis as assessed by quantitative computed tomography (QCT) - 10-year follow-up
}

Mariusz Korkosz ${ }^{1 *}$, Jerzy Gąsowski ${ }^{2}$, Piotr Grzanka ${ }^{3}$, Janusz Gorczowski ${ }^{4}$, Wojciech Pluskiewicz ${ }^{5}$, Sławomir Jeka ${ }^{6}$ and Tomasz Grodzicki ${ }^{7}$

\begin{abstract}
Background: To evaluate the relationship between bone loss and new bone formation in ankylosing spondylitis (AS) using 10-year X-ray, dual-energy x-ray absorptiometry (DXA) and quantitative computed tomography (QCT) follow-up.

Methods: Fifteen AS patients free from medical conditions and drugs affecting bone metabolism underwent X-ray, DXA and QCT in 1999 and 2009.

Results: In spine QCT a statistically significant $(p=0,001)$ decrease of trabecular bone mineral content (BMC) was observed (change \pm SD: $18.0 \pm 7.3 \mathrm{mg} / \mathrm{cm}^{3}$ ). In contrast, spine DXA revealed a significant increase of bone mineral density (change \pm SD: $-0.15 \pm 0.14 \mathrm{~g} / \mathrm{cm}^{2}$ ). The mean BMC, both at baseline and follow-up was significantly lower ( $p=0.02$ and $p=0.005$, respectively) in advanced radiological group as compared to early radiological group. However, in multiple regression model after adjustment for baseline BMC, the baseline radiological scoring did not influence the progression of bone loss as assessed with $Q C T$ ( $p=0.22, p$ for BMC*X-ray syndesmophyte scoring interaction $=0.65, \mathrm{p}$ for ANOVA-based $X$-ray syndesmophyte scoring*time interaction $=0.39$ ). Baseline BMC was the only significant determinant of 10-year BMC change, to date the longest QCT follow-up data in AS.

Conclusions: In AS patients who were not using antiosteoporotic therapy spine trabecular bone density evaluated by QCT decreased over 10-year follow-up and was not related to baseline radiological severity of spine involvement.
\end{abstract}

\section{Background}

Osteoporosis is a well recognized early feature of ankylosing spondylitis (AS) particularly pronounced in active disease [1,2]. In AS axial osteoporosis coexists with new bone formation, ie. syndesmophytes, spine ligaments ossification and facet joints ankylosis, thus the outer layer of the spine is hipermineralized (Figure 1A) as opposed to bone density within vertebral bodies which is diminished. In the effect the risk of vertebral fractures increases [3,4].

\footnotetext{
* Correspondence: mariuszk@mp.pl

'Division of Rheumatology, Department of Internal Medicine and Gerontology, Jagiellonian University, Sniadeckich 10, Krakow, 31-531, Poland Full list of author information is available at the end of the article
}

Bone density in AS is assessed by dual-energy $\mathrm{x}$-ray absorptiometry (DXA) and quantitative computed tomography (QCT). The DXA measures areal bone mineral density (BMD, $\left.\mathrm{g} / \mathrm{cm}^{2}\right)$. Spine anteroposterior DXA reliably detects osteoporosis in early cases but in advanced AS it overestimates BMD due to new bone formation. Spine QCT facilitates measurement of volumetric trabecular bone mineral content $\left(\mathrm{BMC}, \mathrm{mg} / \mathrm{cm}^{3}\right.$ ) with no cortical bone and "outer layer" artifacts. The discrepancy between DXA and QCT in AS is marked in advanced disease, where the majority of patients have normal DXA BMD but QCT shows trabecular bone loss [5].

AS patients with more pronounced new bone formation are at greater risk of osteoporosis [6]. However, to-

\section{C) Biomed Central}




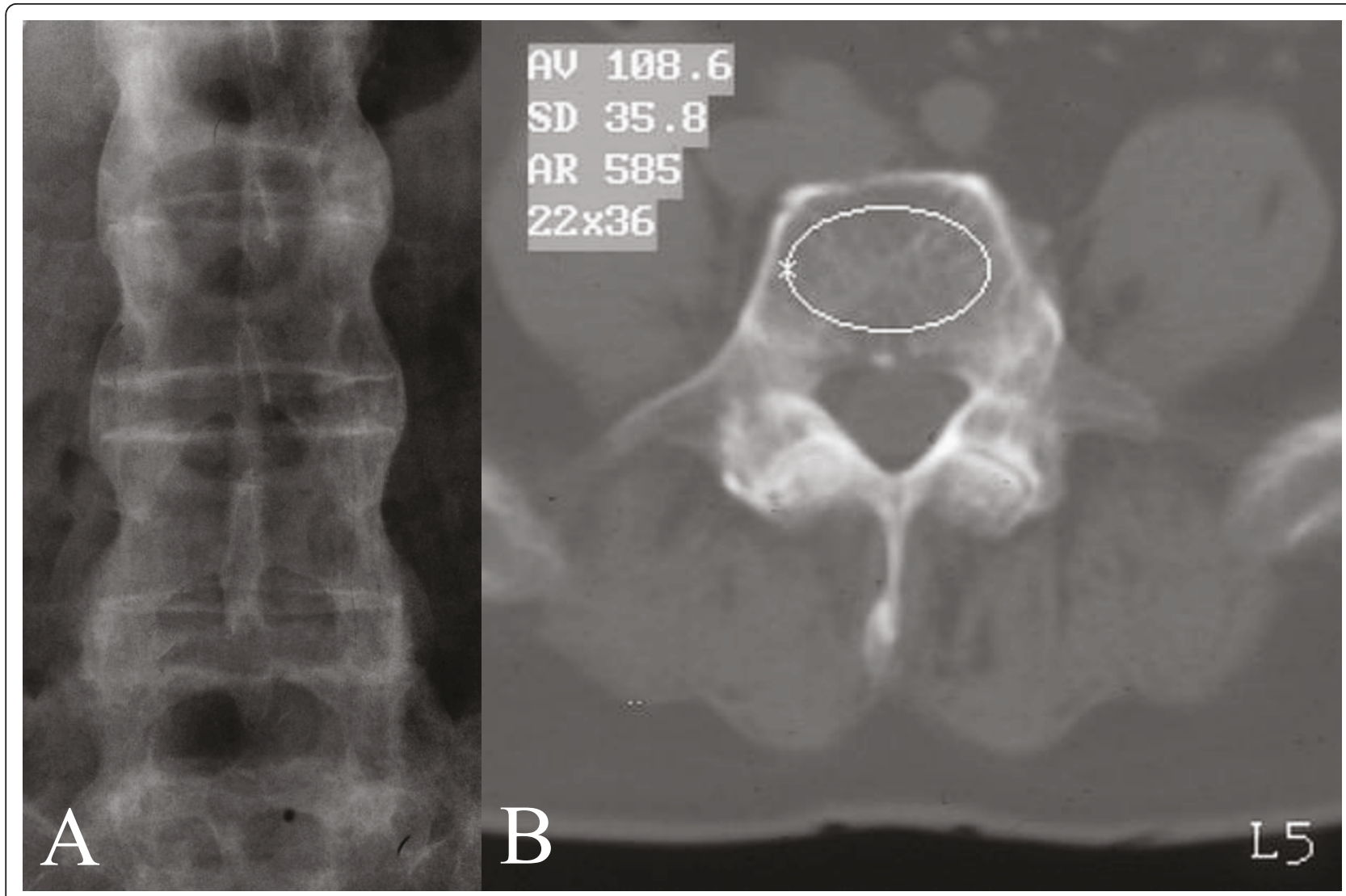

Figure 1 (A) Anteroposterior lumbar radiograph with advanced syndesmophytes and (B) Quantitative Computed Tomography scan of L5 vertebral body showing elliptical region of interest (ROI) containing trabecular bone (48-year-old patient at baseline).

date there is paucity of prognostic data to predict this serious complication.

Therefore the objective of this study was to evaluate the relationship between bone loss and new bone formation in AS using 10-year X-ray, DXA and QCT followup.

\section{Methods}

\section{Patients}

In 1999 thirty seven males fulfilling New York modified AS criteria, with no concomitant diseases and drugs affecting bone metabolism, underwent X-ray (spine), DXA (spine and hip) and QCT (spine) measurements [7]. In 2009 fifteen patients underwent X-ray, DXA and QCT. Of the 22 excluded patients, 14 were treated with bisphosphonates and/or corticosteroids, 3 refused to participate, 1 lost contact. Three patients died. In one patient QCT was not feasible due to unsatisfied positioning.

\section{Imaging and bone density}

We obtained standard radiographs of the lumbar and thoracic spine at baseline and follow-up.
DXA was performed at L2-L4 and left hip using Lunar DPX-IQ (1999) and Lunar Prodigy (2009, Lunar, USA). Right hip was scanned if the left was difficult to position or hip replacement had been performed. In one patient with new L2 and L4 fractures L3 was analyzed. The coefficient of variation (CV) for repeated measurements in vivo and least significant change at $95 \%$ confidence level (spine) were $2,2 \%$ and $0,062 \mathrm{~g} / \mathrm{cm}^{2}$ for DPX-IQ and $1,6 \%$ and $0,043 \mathrm{~g} / \mathrm{cm}^{2}$ for Prodigy, respectively. Tscores and $\mathrm{g} / \mathrm{cm}^{2}$ were based on manufacturer-supplied reference values.

QCT (L1-L5) was determined at baseline and followup with single-energy QCT (Twin Flash, Marconi, USA). Standard calibration phantom (Picker) recommended by Marconi was used for each scan. First, from the lateral lumbar spine scannogram the midportions of L1-L5 vertebral bodies parallel to the endplates were localized manually. Then, scans of $10 \mathrm{~mm}$ thickness were carried out in each vertebrae and other scanning parameters were set according to scanner manufacturer. Data from scanning elliptical region of interest (ROI, Figure $1 \mathrm{~B}$ ) including only trabecular bone, was analyzed using software CirsCT Bone Densitometry of CIRS 
company supplied by scanner manufacturer. T-scores were calculated using manufacturer's reference data. The fractured vertebrae were excluded from analyses.

\section{Statistical analysis}

We compared means using t-test and proportions using chi-square test, where applicable. To check the prognostic value of studied variables on follow-up BMC, we fitted multiple regression models, both without and after adjustment for baseline values of age, body mass index (BMI), erythrocyte sedimentation rate (ESR), smoking, QCT derived BMC stratum, radiologic score and duration of disease prior to baseline visit. To enable visual inspection of individual patient data, we plotted the baseline and followup values of measures of bone-density in strata of baseline BMC based on QCT. To formally test the between-subject (BMC baseline strata) differences in studied variables, we fitted the two-way ANOVA models. Finally, using linear regression approach, we assessed interaction between $\mathrm{BMC}$ and radiographic scoring at baseline, in their influence on measures of bone density at follow-up.

\section{Results}

\section{Patient characteristic}

Mean \pm SD baseline age of 15 included patients was $45.6( \pm 7.3)$ years and disease duration averaged $16.5( \pm$ 8.6) years. ESR averaged $41.8 \mathrm{~mm} / \mathrm{hr}( \pm 27.5)$, BMI 27.4 ( \pm 4.2$), 33,3 \%$ patients were smoking. Three patients experienced four new vertebral fractures during followup (Th7, Th12, L2, L4). Baseline and follow-up densitometry results are shown in table 1 .

\section{Relationship between QCT and DXA results}

We used stratification of patients based on baseline QCT according to Kalender et al. [8] (normal >120; osteopenia
80-120; osteoporosis $<80 ; \mathrm{mg} / \mathrm{cm}^{3}$ ) to follow changes in QCT and DXA. In QCT at baseline there were 4 patients in normal stratum, 5 had osteopenia and 6 osteoporosis. To follow changing bone density data were plotted for individual patients (Figure 2). In spine QCT a statistically significant $(\mathrm{p}=0,001)$ decrease of bone density (change \pm SD: $18.0 \pm 7.3$ ) was observed, and it was universal across all strata (Figure 2). There were no clear-cut trends across strata in DXA that would resemble those noted in spine QCT. In spine DXA, a significant BMD change was noted ( $\mathrm{p}=0,0009)$ with trend towards increased density (change \pm SD: $-0.15 \pm 0.14$ ). Neither neck nor Ward DXA changed from baseline to follow-up. There was no correlation between QCT and DXA, both in density units and T-scores - at baseline and follow-up. Additionally, to test the between-subject (BMC baseline strata) differences in studied variables, we fitted the two-way ANOVA models. We found that the baseline stratification by BMC significantly influenced the $B M C$ values both at baseline $(\mathrm{p}<0.0001)$ and at follow-up $(\mathrm{p}=$ 0.0009 ), but had no impact on the indices derived from DXA (all p > 0.07). We found no interaction between baseline BMC stratum and time-related change in all studied variables (all $\mathrm{p}>0.31$ ).

\section{Relationship between QCT and new bone formation}

To determine the effect of radiological severity on $\mathrm{BMD} / \mathrm{BMC}$ change $\mathrm{X}$-rays were assessed and patients were split into two groups based on baseline syndesmophyte scores according to Devogelaer [9] (table embedded in Figure 3); early group (grade $0-I$, ie. no definite syndesmophytes) - 7 patients; and advanced group (grade II-IV, ie. bridging syndesmophytes) - 8 patients (Figure 3). At follow-up 6 patients progressed into advanced group.

Table 1 Mean DXA and QCT results at baseline and at follow-up

\begin{tabular}{|c|c|c|c|c|c|c|}
\hline & baseline & & follow-up & & change & $\mathbf{p}$ \\
\hline & absolute & T-score & absolute & T-score & & \\
\hline$\overline{\text { QCT L1-L5, mean }\left(\mathrm{mg} / \mathrm{cm}^{3}\right)}$ & 94.2 & -3.654 & 76.1 & -4.468 & 18.0 & $0.001^{*}$ \\
\hline (SD) & 31.8 & 1.246 & 33.5 & 1.499 & & \\
\hline median (25th- $75^{\text {th }}$ percentile) & $96.5(68.2-122.5)$ & & $77.5(47.0-113.7)$ & & & \\
\hline DXA L2-L4, mean $\left(\mathrm{g} / \mathrm{cm}^{2}\right)$ & 1.027 & -1.780 & 1.180 & -0.493 & -0.15 & $0.0009^{*}$ \\
\hline (SD) & 0.183 & 1.531 & 0.198 & 1.649 & & \\
\hline median (25th- $75^{\text {th }}$ percentile) & $0.96(0.86-1.24)$ & & $1.19(1.0-1.35)$ & & & \\
\hline DXA neck, mean $\left(\mathrm{g} / \mathrm{cm}^{2}\right)$ & 0.892 & -1.131 & 0.926 & -1.108 & -0.03 & 0.48 \\
\hline (SD) & 0.134 & 1.077 & 0.279 & 2.139 & & \\
\hline median (25th- $75^{\text {th }}$ percentile) & $0.86(0.83-0.92)$ & & $0.88(0.83-0.96)$ & & & \\
\hline DXA Wards, mean $\left(\mathrm{g} / \mathrm{cm}^{2}\right)$ & 0.737 & -1.715 & 0.727 & -1.792 & 0.01 & 0.81 \\
\hline (SD) & 0.129 & 0.992 & 0.231 & 1.776 & & \\
\hline median (25th- $75^{\text {th }}$ percentile) & $0.73(0.65-0.82)$ & & $0.69(0.62-0.79)$ & & & \\
\hline
\end{tabular}

DXA, dual-energy x-ray absorptiometry; QCT, quantitative computed tomography. 

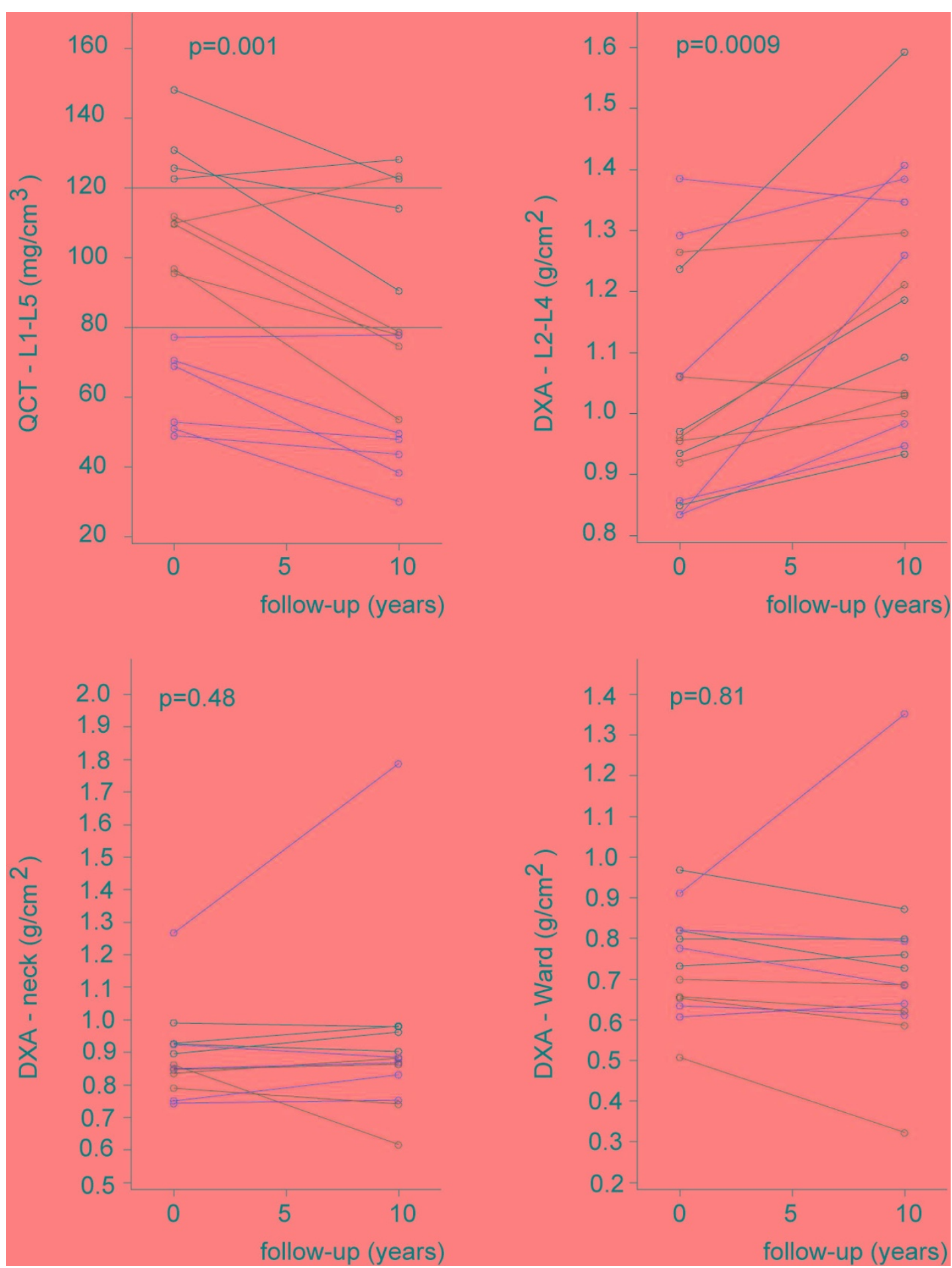

Figure 2 Measures of bone density at baseline and after 10-year follow-up in 15 patients in four regions of interest: L1-L5 (QCT), L2L4 (DXA), neck (DXA) and Wards (DXA) with regard to BMC baseline stratification. Each baseline BMC stratum is represented by different colors: black (normal), green (osteopenia), red (osteoporosis). 


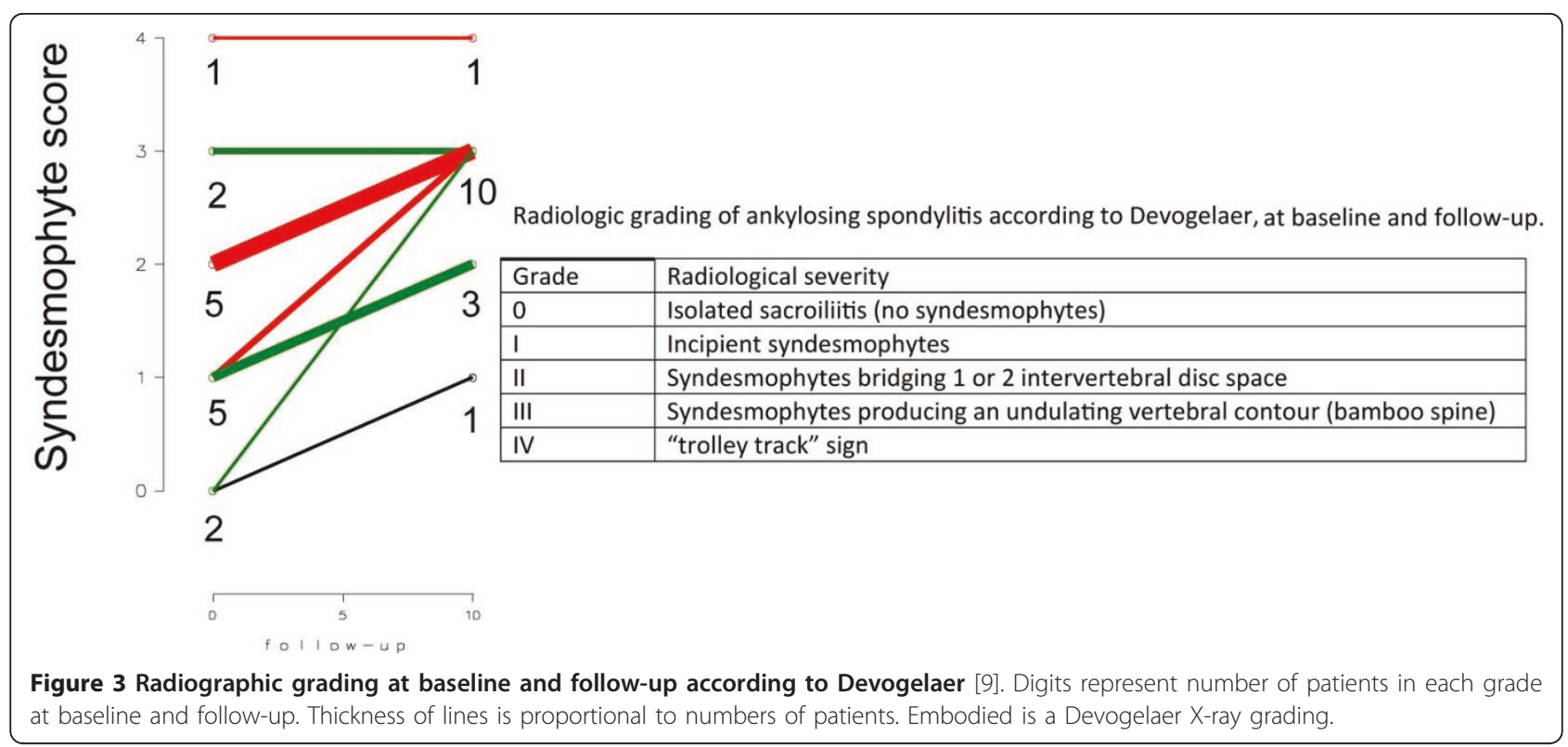

The mean BMC, both at baseline and follow-up was significantly lower $(\mathrm{p}=0.02$ and $\mathrm{p}=0.005$, respectively) in advanced group $\left(77.2 \pm 21.5\right.$ and $55.4 \pm 18.9 \mathrm{mg} / \mathrm{cm}^{3}$, baseline and follow-up, respectively) as compared to early group $\left(113.6 \pm 31.7\right.$ and $99.8 \pm 31.1 \mathrm{mg} / \mathrm{cm}^{3}$, baseline and follow-up, respectively).

However, in multiple regression model after adjustment for baseline $\mathrm{BMC}$, the baseline radiological scoring did not influence the progression of bone loss as assessed with QCT $(\mathrm{p}=0.22$, $\mathrm{p}$ for $\mathrm{BMC} \mathrm{X}$-ray syndesmophyte scoring interaction $=0.65$, $\mathrm{p}$ for ANOVAbased X-ray syndesmophyte scoring"time interaction = 0.39 ).

To discover the predictive value of other variables possibly influencing 10-year changes in BMC similar multiple regression model was used. Only baseline QCT values $\left(\mathrm{mg} / \mathrm{cm}^{3}\right)$ predicted 10-year decrease in QCT; with no influence of baseline DXA values, disease duration, age, ESR, smoking and BMI.

\section{Discussion}

We showed that in patients with ankylosing spondylitis who were free from medical conditions and drugs affecting bone metabolism, spine trabecular bone density evaluated by QCT decreased over 10-year follow-up. Despite of lower BMC in patients with more advanced radiology scoring, the 10 -year change was not related to baseline radiological severity of spine involvement.

Study population was highly homogeneous and included men with no history of steroid or bisphosphonate use over 10-year follow-up. The baseline syndesmophyte formation was equally distributed between early and advanced disease. Thus we have had a group of early new bone formation, ie. scores $0-\mathrm{I}$ corresponding to lack or mild syndesmophyte formation and advanced new bone formation, ie. scores II-IV corresponding to severe bridging osteoproliferation. The higher radiology scores in Devogelaer grading (Figure 3) the more pronounced redistribution of bone, ie. loss of vertebral bodies trabecular bone density is accompanied by an increase in density of the outer layer of spine. This phenomenon seems to be responsible for higher vertebral fracture risk in AS since the osteoporosis within vertebral bodies associated with increased rigidity of spine makes it more vulnerable to fractures. The evolution of these two contrary processes in the natural history of AS is responsible for axial not peripheral fractures $[10,11]$. There is an evidence that different bone density measurements, including spine QCT showed similar percentage differences (range 16-22\%) between subjects with vertebral fractures and controls [12-14] but in AS these differences have not been examined yet.

We showed that QCT is a reliable technique and spine a correct region to follow-up long term changes in $\mathrm{BMC}$, reflecting natural history of osteoporosis in ankylosing spondylitis (Figure 2). Decline in BMC was statistically significant and neither DXA of spine nor femoral neck or Wards revealed a similar trend. In our group, one patient showed an outlying increase in neck and Ward's values (Figure 2). After exclusion of this individual the $\mathrm{p}$ value for change in DXA neck measurement did not change $(p=0.80)$. However we observed a small yet statistically significant $(\mathrm{p}=0.02)$ decrease in DXA measurement of Ward's triangle which represents trabecular bone and is compatible with our QCT findings. This observation strengthens the possible impact of our 
message, that the measurement of trabecular bone (either QCT or DXA Ward's triangle) should replace integral measurement of cortical and trabecular bone (DXA neck or spine) in assessment of AS patients.

In addition, regression analysis determined that the only one significant predictor of the final BMC was baseline BMC. Lee did not show significant changes in BMC over 15-month period, to date the longest published study with QCT in AS [15]. They followed 14 patients; 7 with sacroiliitis alone (early AS) and 7 with extensive vertebral syndesmophytes (late AS) thus with number and strata resembling our group.

Our results suggest that baseline syndesmophytes score has impact on trabecular bone density as early and advanced groups differ regarding QCT values at baseline and follow-up. Karberg using QCT and DXA reported that greater bone loss is detected more frequently in patients with syndesmophytes [6] and our results confirm this as mean BMC in our advanced group was in osteoporosis range both at baseline and at follow-up. In contrast mean BMC in early group was within osteopenia range, both at baseline and follow-up.

Although early and advanced groups differ in QCT values, the baseline radiological severity had no predictive value for bone loss over 10 years. It thus seems likely that in ankylosing spondylitis bone loss does not parallel new bone formation, contrary to findings by Karberg et al. [6].

Our study has to be considered within context of its limitations. The sample-size is relatively low, which restricted our use of statistical techniques to disentangle the effect of possible confounders. The NSAIDs treatment, both continuous and on demand, may have influenced syndesmophyte growth. It is hard to separate the influence of disease and aging on trabecular bone density as well. However, ours is longest follow-up data available in a highly homogenous group of patients. Despite of the relatively small group, we had $>80 \%$ power to detect $20 \%$ difference in the BMC from baseline to follow-up, with the $5 \%$ significance. On the other hand, we are aware, that due to moderately-sized sample size the results we obtained must be considered with caution.

Intriguing question is whether there is a link between bone resorption and new bone formation in AS or whether inflammation triggers both processes. Bone loss is already seen in early AS and is due to disturbed bone turnover rather than immobility caused by syndesmophytes [6,9,15-17]. Markers of bone resorption were found to be positively correlated with ESR and CRP [18-20]. TNF blockade decreased inflammation in AS and increased BMD [21], observations which link inflammation with bone resorption.

Interaction between new bone formation and inflammation in AS is not clear. Osteocalcin was found to be decreased [22], normal $[15,20]$ or increased [23] in AS and was unrelated to levels of inflammatory markers. Most syndesmophytes are not associated with inflammation as based on MRI studies [24]. Anti-TNF treatment in spondyloarthritis increased bone-specific alkaline phosphatase (BALP) and negative correlation between BALP and metalloproteinase-3 (bone destruction marker) was discovered, indicating that new bone formation in AS occurs if inflammation is depressed [25]. Thus it seems likely that bone loss and new bone formation in AS are not directly linked with inflammation, and according to our data they are not coupled - a hypothesis which requires further studies.

\section{Conclusions}

In AS patients who were not using antiosteoporotic therapy spine trabecular bone density evaluated by QCT decreased over 10-year follow-up and was not related to baseline radiological severity of spine involvement.

\section{Abbreviations}

AS: ankylosing spondylitis; DXA: dual-energy x-ray absorptiometry; QCT: quantitative computed tomography; BMD: bone mineral density; BMC: bone mineral content; ROI: region of interest; $\mathrm{CV}$ : coefficient of variation; ESR: erythrocyte sedimentation rate; CRP: C-reactive protein; BMI: body mass index; BALP: bone-specific alkaline phosphatase; TNF: tumor necrosis factor; NSAIDs: nonsteroidal anti-inflammatory drugs.

\section{Author details}

'Division of Rheumatology, Department of Internal Medicine and Gerontology, Jagiellonian University, Sniadeckich 10, Krakow, 31-531, Poland. ${ }^{2}$ Department of Internal Medicine and Gerontology, Jagiellonian University, Sniadeckich 10, Krakow, 31-531, Poland. ${ }^{3}$ Imaging Unit, Department of Medicine, Jagiellonian University, Skawinska 8, Krakow, 31-066, Poland. ${ }^{4}$ Densitometry Unit, Malopolskie Centrum Medyczne, Rejtana 2, Krakow, 30510, Poland. ${ }^{5}$ Metabolic Bone Diseases Unit, Medical University of Silesia, 3Maja 13-15, Zabrze, 41-800, Poland. 'Department of Rheumatology and Connective Tissue Diseases, $2^{\text {nd }}$ University Hospital, Ujejskiego 75, Bydgoszcz, 85-168, Poland. 'Department of Internal Medicine and Gerontology, Jagiellonian University, Sniadeckich 10, Krakow, 31-531, Poland.

\section{Authors' contributions}

MK was responsible for study concept and design, patients assessment, analysis of investigated data and its interpretation, and drafting manuscript. JG performed statistical analysis, participated in data analysis and interpretation, figures preparation and drafting manuscript. PG was responsible for QCT scanning and analysis, and quality control of data. JG carried out DXA scanning and analysis, and quality of data. WP participated in study concept and design, DXA and QCT quality control, and data interpretation. SJ was involved in analysis of X-ray, DXA and QCT data, and statistical analysis. TG was responsible for study concept and revising manuscript. All authors read and approved the final manuscript.

\section{Competing interests}

The authors declare that they have no competing interests.

Received: 22 October 2010 Accepted: 31 May 2011

Published: 31 May 2011

\section{References}

1. Gratacos J, Collado A, Pons F, Osaba M, Sanmarti R, Roque M, et al: Significant loss of bone mass in patients with early, active ankylosing spondylitis: A followup study. Arthritis Rheum 1999, 42(11):2319-24. 
2. Maillefert JF, Aho LS, El Maghraoui A, Dougados M, Roux C: Changes in bone density in patients with ankylosing spondylitis: A two-year followup study. Osteoporos Int 2001, 12(7):605-9.

3. Donnelly S, Doyle DV, Denton A, Rolfe I, McCloskey EV, Spector TD: Bone mineral density and vertebral compression fracture rates in ankylosing spondylitis. Ann Rheum Dis 1994, 53(2):117-21.

4. Mitra D, Elvins DM, Speden DJ, Collins AJ: The prevalence of vertebral fractures in mild ankylosing spondylitis and their relationship to bone mineral density. Rheumatology (Oxford) 2000, 39(1):85-9.

5. Lange U, Kluge A, Strunk J: Ankylosing spondylitis and bone mineral density - what is the ideal tool for measurement? Rheumatol Int 2005, 26:115-20.

6. Karberg K, Zochling J, Sieper J, Felsenberg D, Braun J: Bone loss is detected more frequently in patients with ankylosing spondylitis with syndesmophytes. J Rheumatol 2005, 32(7):1290-8.

7. Korkosz M, Gluszko P, Marcinek P: Comment on bone mineral density and bone turnover markers in a group of male ankylosing spondylitis patients. J Clin Rheumatol 2002, 8(6):359-60.

8. Kalender WA, Felsenberg D, Louis O, Lopez P, Klotz E, Osteaux M, et al: Reference values for trabecular and cortical vertebral bone density in single and dual-energy quantitative computed tomography. Eur J Radiol 1989, 9(2):75-80

9. Devogelaer JP, Maldague B, Malghem J, Nagant de Deuxchaisnes C: Appendicular and vertebral bone mass in ankylosing spondylitis. A comparison of plain radiographs with single- and dual-photon absorptiometry and with quantitative computed tomography. Arthritis Rheum 1992, 35(9):1062-7.

10. Cooper C, Carbone L, Michet CJ, Atkinson EJ, O'Fallon WM, Melton LJ: Fracture risk in patients with ankylosing spondylitis: A population based study. J Rheumatol 1994, 21(10):1877-82.

11. Vosse D, Landewe R, van der Heijde D, van der Linden S, van Staa TP, Geusens P: Ankylosing spondylitis and the risk of fracture: Results from a large primary care-based nested case-control study. Ann Rheum Dis 2009, 68(12):1839-42.

12. Grampp S, Genant HK, Mathur A, Lang P, Jergas M, Takada M, et al: Comparisons of noninvasive bone mineral measurements in assessing age-related loss, fracture discrimination, and diagnostic classification. J Bone Miner Res 1997, 12(5):697-711.

13. Kroger H, Lunt M, Reeve J, Dequeker J, Adams JE, Birkenhager JC, et al: Bone density reduction in various measurement sites in men and women with osteoporotic fractures of spine and hip: The european quantitation of osteoporosis study. Calcif Tissue Int 1999, 64(3):191-9.

14. Lang TF, Guglielmi G, van Kuijk C, De Serio A, Cammisa M, Genant HK Measurement of bone mineral density at the spine and proximal femur by volumetric quantitative computed tomography and dual-energy $\mathrm{X}$ ray absorptiometry in elderly women with and without vertebral fractures. Bone 2002, 30(1):247-50.

15. Lee YS, Schlotzhauer T, Ott SM, van Vollenhoven RF, Hunter J, Shapiro J, et al: Skeletal status of men with early and late ankylosing spondylitis. Am J Med 1997, 103(3):233-41.

16. Mullaji AB, Upadhyay SS, Ho EK: Bone mineral density in ankylosing spondylitis. DEXA comparison of control subjects with mild and advanced cases. J Bone Joint Surg Br 1994, 76(4):660-5.

17. Will R, Palmer R, Bhalla AK, Ring F, Calin A: Osteoporosis in early ankylosing spondylitis: A primary pathological event? Lancet 1989, 23 30;2(8678-8679):1483-5.

18. El Maghraoui A, Borderie D, Cherruau B, Edouard R, Dougados M, Roux C: Osteoporosis, body composition, and bone turnover in ankylosing spondylitis. J Rheumatol 1999, 26(10):2205-9.

19. Lange U, Teichmann J, Strunk J, Muller-Ladner U, Schmidt KL: Association of 1.25 vitamin D3 deficiency, disease activity and low bone mass in ankylosing spondylitis. Osteoporos Int 2005, 16(12):1999-2004.

20. Toussirot E, Ricard-Blum S, Dumoulin G, Cedoz JP, Wendling D: Relationship between urinary pyridinium cross-links, disease activity and disease subsets of ankylosing spondylitis. Rheumatology (Oxford) 1999, 38(1):21-7.

21. Allali F, Breban M, Porcher R, Maillefert JF, Dougados M, Roux C: Increase in bone mineral density of patients with spondyloarthropathy treated with anti-tumour necrosis factor alpha. Ann Rheum Dis 2003, 62(4):347-9.
22. Mitra D, Elvins DM, Collins AJ: Biochemical markers of bone metabolism in mild ankylosing spondylitis and their relationship with bone mineral density and vertebral fractures. J Rheumatol 1999, 26(10):2201-4.

23. Grisar J, Bernecker PM, Aringer M, Redlich K, Sedlak M, Wolozcszuk W, et al: Ankylosing spondylitis, psoriatic arthritis, and reactive arthritis show increased bone resorption, but differ with regard to bone formation. J Rheumatol 2002, 29(7):1430-6.

24. Sieper J: Can structural damage be prevented in ankylosing spondylitis? Curr Opin Rheumatol 2009, 21(4):335-9.

25. Appel H, Janssen L, Listing J, Heydrich R, Rudwaleit M, Sieper J: Serum levels of biomarkers of bone and cartilage destruction and new bone formation in different cohorts of patients with axial spondyloarthritis with and without tumor necrosis factor-alpha blocker treatment. Arthritis Res Ther 2008, 10(5):R125.

\section{Pre-publication history}

The pre-publication history for this paper can be accessed here: http://www.biomedcentral.com/1471-2474/12/121/prepub

doi:10.1186/1471-2474-12-121

Cite this article as: Korkosz et al:: Baseline new bone formation does not predict bone loss in ankylosing spondylitis as assessed by quantitative computed tomography (QCT) - 10-year follow-up. BMC Musculoskeletal Disorders 2011 12:121.

\section{Submit your next manuscript to BioMed Central and take full advantage of:}

- Convenient online submission

- Thorough peer review

- No space constraints or color figure charges

- Immediate publication on acceptance

- Inclusion in PubMed, CAS, Scopus and Google Scholar

- Research which is freely available for redistribution

Submit your manuscript at www.biomedcentral.com/submit
C Biomed Central 